PSICOLOGÍA

IBEROAMERICANA
Psicología Iberoamericana ISSN: 1405-0943

revista.psicologia@ibero.mx

Universidad Iberoamericana, Ciudad de México México

\title{
Impacto de la telepsicología en la satisfacción de la atención a pacientes con Covid-19
}

Argüero Fonseca, Aimée; Aguirre Ojeda, Diana Patricia; Reynoso González, Oscar Ulises; Girón Pérez, Manuel Iván; Espinosa Parra, Irene Margarita; Sierra Murguía, Mariana Alejandra Impacto de la telepsicología en la satisfacción de la atención a pacientes con Covid-19

Psicología Iberoamericana, vol. 29, núm. 3, Esp., e293325, 2021

Universidad Iberoamericana, Ciudad de México, México

Disponible en: https://www.redalyc.org/articulo.oa? $\mathrm{id}=133968747007$

DOI: https://doi.org/10.48102/pi.v29i3.325

\section{(c) $(1)$}

Esta obra está bajo una Licencia Creative Commons Atribución 4.0 Internacional. 
Psicología Iberoamericana, vol. 29, núm. 3, Esp., e293325, 2021

Universidad Iberoamericana, Ciudad de México, México

Recepción: 25 Enero 2021

Aprobación: 05 Agosto 2021

DOI: https://doi.org/10.48102/ pi.v29i3.325

Redalyc: https://www.redalyc.org/ articulo.oa?id $=133968747007$
Artículos

\section{Impacto de la telepsicología en la satisfacción de la atención a pacientes con Covid-19}

Impact of telepsychology on care satisfaction for patients with

COVID-19

Aimée Argüero Fonseca aimee.arguero@uan.edu.mx Universidad Autónoma de Nayarit, México

(iD https://orcid.org/0000-0002-3864-5299

Diana Patricia Aguirre Ojeda diana.aguirre@uan.edu.mx Universidad Autónoma de Nayarit, México

(D) https://orcid.org/0000-0003-1814-5534 Oscar Ulises Reynoso González ulises.reynoso@academicos.udg.mx Universidad de Guadalajara, México https://orcid.org/0000-0002-0598-4665 Manuel Iván Girón Pérez ivangiron@uan.edu.mx Universidad Autónoma de Nayarit, México

iD https://orcid.org/0000-0001-6808-6590

Irene Margarita Espinosa Parra irene.parra@uan.edu.mx Universidad Autónoma de Nayarit, México

iD https://orcid.org/0000-0003-0576-9356 Mariana Alejandra Sierra Murguía msierra@cancercentertec100.com Cancer Center Tec100, México

https://orcid.org/0000-0001-5806-0688

Resumen: La COVID-19 presentó retos para la psicoterapia, por lo cual es pertinente realizar estudios que valoren la opinión de los usuarios respecto al uso de telepsicología, para determinar su eficiencia. El presente estudio preliminar, se realizó con un diseño de un solo grupo y una medida. Se aplicó la escala de satisfacción con el tratamiento recibido (CRES-4) a 61 nayaritas, positivos de COVID-19 (Mujeres=41; Hombres=20), con edades entre 16 y 67 años $(\mathrm{ME}=36$; $\mathrm{DE}=8.13)$, derivados de un laboratorio de detección, que recibieron acompañamiento telepsicológico usando mindfulness. Para comparar la satisfacción con el tratamiento, se realizó un conjunto de pruebas $U$ de Mann Whitney y el estado emocional con la prueba de rangos de Wilcoxon, considerando el tamaño del efecto con la $\mathrm{r}$ de Rosenthal. De lo anterior, resultó una puntuación global alta $(\mathrm{ME}=81.88 ; \mathrm{DE}=17.96)$, que sugiere que la evaluación es satisfactoria e influye efectivamente en la mejora del estado emocional. Se sugiere incorporar muestras más representativas. Con todo, los resultados obtenidos permiten evaluar a grandes rasgos la percepción de la eficacia de estas intervenciones.

Palabras clave: Telepsicología, satisfacción en la atención, Covid-19, ética en la teleterapia, mindfulness. 


\begin{abstract}
COVID-19 presented challenges for psychotherapy, so it is pertinent to carry out studies that assess the opinion of users regarding the use of telepsychology, to determine its efficiency. The present preliminary study was carried out with a single group and one measure design. The satisfaction scale with the treatment received (CRES-4) was used to test 61 participants from Nayarit. Participants were positive for COVID-19 (Women $=41 ;$ Men $=20)$, aged between 16 and 67 years $(\mathrm{ME}=36 ; \mathrm{SD}=$ 8.13). The sample was from a COVID-19 detection laboratory and participants received telepsychological monitoring using mindfulness techniques. To compare satisfaction with treatment, a set of Mann Whitney U tests on their emotional state were performed with the Wilcoxon rank test, considering the effect size with Rosenthal's r. From the above, a high global score resulted $(\mathrm{ME}=81.88$; $\mathrm{SD}=17.96)$, which suggests that the evaluation is satisfactory and effectively influences the improvement of the emotional state. It is suggested to incorporate more representative samples. Despite this, the results obtained allow a broad assessment of the perception of the efficacy of these interventions.
\end{abstract}

Keywords: telepsychology, Covid-19, care satisfaction, ethics and telatherapy, mindfulness.

\title{
Introducción
}

La pandemia por COVID-19 se ha convertido en una de las prioridades que deben abordarse urgentemente pues es una amenaza de amplia proporción para el bienestar de sociedades enteras que han sido afectadas gravemente por esta crisis. La adversidad relacionada con las repercusiones socioeconómicas, el miedo a la propagación del virus, así como las preocupaciones asociadas, impactan sin duda alguna en la salud tanto física como mental de la población (Organización Panamericana de la Salud [OPS], 2020).

A nivel mundial se han notificado 2,880,726 muertes a causa de este virus, mientras que en México han fallecido 205,002 (Organización Mundial de la Salud [OMS], 2021). En este país, la situación se ha vuelto extremadamente compleja pues los casos de personas con COVID-19 y los fallecimientos siguen aumentando. Esto implica un impacto económico y social negativo y provoca confusión con respecto al mantenimiento de las medidas como la sana distancia para evitar la transmisión en lugares públicos y de trabajo (OPS \& OMS México, s/f).

Asimismo, datos de la OMS (2020) afirman que la pandemia ha provocado un incremento en la demanda de servicios de salud mental. Muchas personas han aumentado su consumo de alcohol o drogas y padecen crecientes problemas de ansiedad e insomnio. Los trastornos mentales se han agravado a causa del duelo, el aislamiento, la pérdida de ingresos y el miedo; además de que la COVID-19 por sí misma puede traer complicaciones neuropsicológicas como delirios, agitación o eventos cerebrovasculares.

Por lo anterior, políticas de Organización de las Naciones Unidas (ONU) (2020) para minimizar los efectos de la pandemia en la salud mental, enfatizan que es importante considerar urgentemente tres medidas recomendadas. La primera de ellas es aplicar un enfoque que abarque a toda la sociedad para promover, proteger y cuidar la salud mental. La segunda, garantizar la disponibilidad amplia de apoyo 
psicosocial y en materia de salud mental de emergencia. Por último, apoyar la respuesta a la COVID-19 creando servicios de salud mental para el futuro.

Como se puede observar los servicios de salud mental durante la pandemia por COVID-19 deben estar disponibles en cualquier emergencia. Sin embargo; dar atención psicológica durante la COVID-19 no es sencillo, seguir las precauciones universales para mitigar la propagación de este virus tales como las prácticas de higiene, quedarse en casa y el distanciamiento social, dificulta la atención presencial por lo que se requiere de la implementación de nuevas formas de intervención a distancia.

Ante ello, la Asociación Americana de Psicología (American Psychological Association, 2013) refiere que los profesionales de la psicología que proporcionen servicios presenciales deben estar preparados para situaciones emergentes, adoptando las medidas necesarias para garantizar su competencia y desarrollar nuevos conocimientos y habilidades que les permitan brindar una práctica eficaz en el manejo de riesgos de manera remota.

Es aquí donde entra la telepsicología, una herramienta que puede utilizarse como complemento o sustitución de los servicios presenciales. La telepsicología se define como la atención psicológica que utiliza tecnologías de la información y de la telecomunicación a través del procesamiento de la información por dispositivos electrónicos, electromagnéticos, electro-ópticos, electromecánicos y eléctricos (American Psychological Association, 2013), tales como los aparatos móviles, teléfonos, videoconferencias, blogs, webs de autoayuda, redes sociales, que facilitan la comunicación sincrónica y por medio del correo electrónico, que facilitan la comunicación asíncrona.

\section{Telepsicología}

La telepsicología tiene su origen en los cambios tecnológicos que facilitan el acceso a poblaciones diversas. Sin embargo, no todas las naciones han podido alcanzar esta modalidad asistencial, debido a que se necesita que al mismo tiempo exista un grado de desarrollo tecnológico avanzado en las mismas (Colegio Oficial de Psicólogos de Madrid, 2017).

La telepsicología es geográficamente flexible y tiene el potencial de revolucionar los servicios psicológicos en locaciones remotas o con dificultades de movilidad y de dar atención a quienes prefieran el acceso a los servicios de una manera más accesible. Se espera que los psicólogos cuenten con los componentes básicos para usar las novedades tecnológicas en su práctica como el entendimiento de conceptos relacionados al procesamiento de datos, almacenamiento electrónico y la infraestructura del internet (Gamble et al., 2015).

Además, el uso de las tecnologías aplicadas en la psicología implica un reto para los profesionales sobre cuestiones jurídicas, deontológicas y clínicas que se deben de manejar con mucho cuidado ya que se asumen riesgos y responsabilidades importantes de los que se debe ser 
consciente, por ejemplo la identificación, veracidad de datos, privacidad, confidencialidad, jurisdicción, calidad técnica y el afrontamiento de situaciones emergentes (American Psychological Association, 2013).

\section{Ventajas y desventajas de la telepsicología}

La guía para la práctica de la telepsicología que publicó el Consejo General de la Psicología de España (2017), menciona que la telepsicología tiene ventajas tanto para quien la lleva a cabo (profesional), así como para quien la recibe (pacientes/clientes). Algunas de las ventajas que se destacan en la telepsicología son el acercamiento a personas que no pueden trasladarse y la proximidad en las fronteras y regiones.

No obstante, esta guía también considera que la telepsicología enfrenta retos o desventajas que son necesarias considerar en todas las etapas y fases del tratamiento en los profesionales y el paciente, entre las que se encuentran aspectos de privacidad, el carácter confidencial y hacer frente a demandas o situaciones de urgencia.

Por otra parte, Acero et al. (2020) refieren que la utilización de la tecnología en los programas de tratamientos en línea tiene grandes ventajas que permiten apegarse a los lineamientos éticos de la profesión, teniendo resultados útiles y de largo alcance para las personas que se encuentran en algún tipo de situación de riesgo y les es complicado desplazarse a otro sitio.

Asimismo, Acero et al. (2020) mencionan que la telepsicología permite que los pacientes tengan un rápido acceso al servicio de atención sin que el horario sea un inconveniente debido a que se permanece en casa y es posible la conexión con el profesional en un contexto de mutuo acuerdo. Los autores también consideran que la telepsicología proporciona la ventaja de no tener que enfrentarse el paciente a obstáculos de estigmatización en las clínicas de atención mental y psicológica que llegan a limitar la identificación de problemáticas y su tratamiento.

McDonald et al. (2019) examinaron el uso pionero de la atención psicológica autónoma mediante programas de psicoterapia que pueden mejorar en gran parte el acceso a los servicios de nivel individual y poblacional, encontrando pruebas sólidas que respaldan la terapia cognitivo-conductual en línea.

Por otro lado, algunas de las inquietudes que se pueden convertir en desventajas en el quehacer de la telepsicología de acuerdo con Richards y Richardson (2012, citado en Acero et al., 2020), está relacionada con el manejo de situaciones de crisis y aspectos técnicos centrados en el dominio de las tecnologías y el adecuado uso de las plataformas, aunado a los problemas de conectividad que pueden llegar a interferir el proceso de comunicación.

En ese sentido, De la Torre y Pardo (2019) refieren que las situaciones que inquietan a los profesionales de la psicología en la intervención telepsicología es el no tener al alcance la información no verbal de los pacientes, lograr establecer un vínculo de confianza y el resguardo de la información proporcionada. 
Algunas otras limitaciones de la atención telepsicológica referidas por González y Sánchez (2020) son las condiciones concernientes a la infraestructura de los hogares lo cual llega a afectar la privacidad de la información, así como las fallas que se pueden derivar de las conexiones de internet y que además no todas las personas tienen acceso a las tecnologías por cuestiones económicas y carencia de servicios.

De la Torre y Pardo (2019) mencionan que no se recomienda el servicio de atención telepsicológica en situaciones de crisis o condiciones específicas como la falta de control emocional, características de personas con trastorno psicótico, depresión grave, situaciones de violencia grave, abuso, entre otras; ya que requiere un abordaje específico y en algunos casos de un equipo multidisciplinar.

En un punto intermedio, Fejit et al. (2020) encontraron tanto ventajas como desventajas sobre las experiencias de los profesionales al brindar atención de salud mental en línea durante la pandemia COVID-19. Sus hallazgos revelan que dentro de las desventajas están los problemas en la tecnología y usabilidad, las dificultades para crear una relación con los usuarios y que no todos los problemas de salud mental y los tratamientos son igual de susceptibles de interacción en línea. En cuanto a las ventajas, los médicos encuestados mostraron ser positivos sobre la efectividad del tratamiento, la flexibilidad y el tiempo de viaje requerido.

\section{Manuales de telepsicología}

Existen diversos manuales para la práctica de la telepsicología, uno de ellos es el de la American Psychological Association (2013) llamado "Guía para la práctica de la Telepsicología" creada con la finalidad de brindar recomendaciones con base a los Principios Éticos y Deontológicos a los profesionales de la psicología en todos sus campos (clínico, de salud, laboral, deportivo, social, educativo, legal, etc.). Otra guía es la del Centro de Psicología Aplicada de la Universidad Autónoma de Madrid en Colaboración con el Colegio Oficial de Psicólogos de Madrid (2018), que presenta una serie de indicaciones para ayudar en la toma de decisiones de los psicólogos en su práctica clínica.

En Colombia, Acero et al. (2020, p. 12) hicieron un documento llamado "Telepsicología: sugerencias para la formación y el desempeño profesional responsable" enfocada en los aspectos relacionados con la prestación de servicios en psicología clínica. Específicamente sobre el COVID, La Comisión Nacional de Salud de China fue pionera en desarrollar guías para las autoridades locales para promover intervenciones psicológicas en crisis para pacientes, personal sanitario y gente bajo observación médica instalando una "hotline" para la salud mental con un panel de intervenciones en crisis por la epidemia que consistía en 73 psicólogos y psiquiatras (Comisión Nacional de Salud de las Personas de República de China, 2020).

En Madrid, el Colegio Oficial de la Psicología de Madrid en colaboración con los equipos de la Clínica Universitaria de Psicología UCM, PsiCall UCM y profesores del Departamento de Psicología 
Clínica de la Universidad Complutense de Madrid, publicó la Guía para el abordaje no presencial de las consecuencias psicológicas del brote epidémico de COVID-19 en la población general.

Dicha Guía está "destinada a profesionales de la Psicología con habilitación para el ejercicio sanitario y formación específica que pretendan intervenir, por medios telemáticos, con población general afectada por el aislamiento y otros fenómenos psicológicos asociados a la epidemia y sus consecuencias" (Larroy et al., 2020, p. 6).

En el 2020, en México, la Secretaría de Salud junto con Martínez, Andrade y Vieyra (del Programa de Intervención en Crisis a Víctimas de Desastres Naturales y Socio organizativos de la Facultad de Psicología de la Universidad Nacional Autónoma de México); Esquivias (del Departamento de Educación Continua del Instituto Nacional de Psiquiatría Ramón de la Fuente Muñiz); Merlín (de la Dirección de Servicios Clínicos del Instituto Nacional de Psiquiatría Ramón de la Fuente Muñiz); Neria (del Instituto Mexicano de Psicoterapia Cognitivo Conductual); Bezanilla (de Psicólogos Sin Fronteras México A.C) y el Centro de Intervención en Crisis, elaboraron el "Manual operativo del Curso Emergente para la Brigada de Atención Psicoemocional y Psicosocial a distancia durante la pandemia de la COVID-19 en México".

En Nayarit, Argüero-Fonseca et al. (2020), elaboraron la "Guía de atención psicológica virtual para personas de grupos vulnerables en crisis por la pandemia COVID-19”. Esta guía fue diseñada como una estrategia educativa para que los psicólogos realicen intervenciones a distancia con la finalidad de brindar atención psicológica a los pacientes afectados, su familia y la sociedad en general.

Las guías mencionadas proporcionan a los psicólogos las recomendaciones necesarias para las buenas prácticas de la telepsicología. En los siguientes párrafos se explica cómo han sido aplicados estos fundamentos para la atención de personas afectadas psicoemocionalmente de manera específica por la pandemia COVID-19.

\section{Telepsicología durante la COVID-19}

Debido a los problemas mentales causados por la pandemia, los investigadores han hecho énfasis en la importancia del tratamiento psicológico, sin embargo, la intervención presencial se ha visto obstaculizada a causa de las medidas necesarias para mitigar la propagación de la COVID-19 que hacen un llamado a quedarse en casa y mantener distancia social.

La rápida transmisión entre las personas de la COVID-19, hace difícil las intervenciones psicológicas tradicionales cara a cara. La popularización de los servicios de internet, los celulares y el surgimiento de redes móviles de quinta generación (5G), han facilitado la disponibilidad de los servicios de salud mental en línea durante la pandemia (Liu et al. 2020).

Cuando la OMS anunció el brote de coronavirus como una pandemia, los médicos tuvieron que enfrentarse al desafío de continuar con la atención. La teleterapia se volvió el medio principal de prestación de 
servicios para la mayoría de quienes nunca la habían usado o habían hecho uso moderado de ésta. Incluso, organizaciones como el Instituto de la Familia de la Universidad de Northwestern integraron la práctica de la telerapia al $100 \%$ para brindar terapia de pareja y familiar (Burgoyne $\&$ Cohn, 2020).

En una revisión sistemática sobre la telepsicología en la pandemia Covid-19 en donde se incluyeron los estudios que utilizaban esta herramienta para tratar pacientes, los resultados respaldaron la efectividad del uso de la telepsicología en el tratamiento de la depresión, ansiedad y estrés (Argüero-Fonseca et al., 2021).

Pierce et al. (2021) reclutaron a 2,619 psicólogos estadounidenses con la finalidad de documentar cómo la pandemia por COVID-19 ha alterado la atención de la salud mental y encontraron que el uso de la telepsicología que estos profesionales hacían antes de la pandemia se multiplicó del $7,07 \%$ al $85,53 \%$ durante la pandemia, porcentaje del cual el $67,32 \%$ realizaban toda su labor clínica con telepsicología. También descubrieron un aumento de más de 26 veces en el uso de la telepsicología en centros de tratamiento para pacientes ambulatorios y de siete veces en centros médicos de Asuntos de Veteranos. Igualmente, hubo un mayor aumento en el porcentaje del uso de la telepsicología en mujeres, en psicólogos que refirieron un aumento en capacitación sobre telepsicología y políticas organizaciones de apoyo, y en psicólogos que atendieron problemas de relaciones, ansiedad y problemas de mujeres. Sin embargo, aunque se produjo un aumento notable en el uso de la telepsicología en tiempos de pandemia, las características individuales y de la práctica afectaron la capacidad de los psicólogos para adoptarla.

De acuerdo con el Colegio Oficial de Psicología de Madrid et al. (2020), la necesidad de utilizar servicios telepsicológicos durante la COVID-19 surge de cuatro aspectos. La imposibilidad de desarrollar intervenciones cara a cara, la escala personal y temporal del fenómeno, la dimensión de Salud Pública y la limitada base de evidencia disponible. Por lo anterior, se deben considerar un conjunto de principios generales al abordar las alteraciones derivadas de esta enfermedad. Algunos de ellos son generar comprensión, generar alivio, promover el equilibrio entre extremos y aumentar recursos de afrontamiento.

Tullio et al. (2020) mencionan que en Italia muchos profesionales no estaban preparados, metodológicamente y tecnológicamente, para manejar el repentino cambio de las terapias tradicionales a las terapias digitales cuando se enfrentaron a la pandemia de COVID-19. En su investigación refieren que los aspectos críticos destacados por esta emergencia sanitaria giran en torno a cuestiones teóricas y de aplicación de la práctica en línea relacionados a temas deontológicos, legales y éticos. Aspectos que los autores plantean, deben abordarse cuidadosamente con el fin de garantizar a los pacientes, la asistencia adecuada y promover la continuación de la atención en línea, una vez terminada la pandemia.

En los países bajos, Fejit et al. (2020) llevaron a cabo una encuesta en línea dirigida a profesionales de la salud para comprender las implicaciones del cambio inesperado en la prestación de atención de 
salud mental presencial a medios en línea, encontrando como principales desafíos los problemas tecnológicos y de usabilidad, así como las dificultades para establecer un vínculo con los clientes.

Por otra parte, es importante reconocer que la pandemia COVID-19 ha generado una serie de cambios en diferentes esferas de la vida de las personas que ha impactado en todas las etapas del ciclo vital, desde los niños y niñas hasta los adultos mayores, en las que es posible que se generen crisis que lleguen a repercutir en la salud física y emocional de las familias. Balluerka et al. (2020) mencionan en un informe de investigación titulado, Las consecuencias psicológicas de la Covid 19 y el confinamiento, que la condición de confinamiento y lo que trae consigo una pandemia detona diferentes situaciones de estrés, provocando miedos e inestabilidad emocional que llegan a afectar la vida de las personas y de su entorno.

Respecto a los factores de vulnerabilidad psicopatológica en una población, Balluerka et al. (2020) refieren que algunas personas son más propensas a tener afectaciones por las condiciones variables de edad, estado mental y psicológico, condiciones físicas, económicas, educativas y de aislamiento, entre otras minorías a las que es necesario prestar atención y prevenir mayores afectaciones en el individuo. Con base a lo anterior, Guerrero (2020), menciona las principales situaciones que se pueden desencadenar a partir de la pandemia son:

\section{Cuarentena}

El periodo de confinamiento ha generado una estadía prolongada dentro de los hogares, en espacios cerrados y en contacto permanente con otros miembros de la familia, donde se labora, se estudia, se convive y se realizan múltiples actividades al mismo tiempo.

\section{Salud}

Las afectaciones físicas y emocionales son el resultado de incapacidades o complicaciones dentro del grupo familiar que afecta a todos los integrantes.

\section{Muertes}

El riesgo de muerte de familiares o personas cercanas ante el contagio en muchas de las ocasiones con la imposibilidad de afrontar el duelo.

\section{Economia}

Algunas familias experimentan pérdidas económicas importantes en el grupo familiar generado por el desempleo, la falta de actividad económica o la inversión en los tratamientos ante la enfermedad. 


\section{Crisis social}

En este periodo la pandemia ha generado crisis sociales importantes desde el desabastecimiento, pánico colectivo, saqueos, incertidumbre gubernamental y educativa que impactan en cada uno de los estratos sociales.

Como se puede observar la pandemia afecta diferentes esferas significativas de la vida lo que implica la importancia de saber en qué momentos usar los servicios de telepsicología al igual que saber cómo se debe llevar a cabo y qué consideraciones o normas se deben de tener en cuenta para su aplicación, por lo tanto, a continuación, se mencionan las cuestiones éticas para la práctica eficaz de la telepsicología.

\section{Estándares éticos de atención en la prestación de servicios de la telepsicología}

La Asociación Americana de Psicología (American Psychological Association, 2013) propone una "Guía para la práctica de la telepsicología: entrenamiento, estándares éticos y requerimientos legales” que es necesaria considerar para la correcta aplicación de la telepsicología ante COVID-19 la cual está enfocada al diseño de directrices de atención telepsicológica dirigida a psicólogos y psicólogas.

Dentro de estos estándares éticos en la práctica de la telepsicología, la American Psychological Association (2013) contempla que el profesional tenga la competencia profesional, cuide los aspectos legales, lleve a cabo el código ético que rige la práctica del profesional de la psicología, cuente con conocimientos en el uso de las tecnologías, así como de las políticas y restricciones institucionales.

La American Psychological Association (2013) determinó también que otro aspecto a considerar es la alianza y contrato terapéutico. Éstos deberán considerar todos los aspectos multiculturales del lugar de origen del paciente y deben aplicarse en la intervención con un estricto apego a los lineamientos éticos, por ser un ámbito nuevo en el que una inadecuada práctica puede llevar a múltiples riesgos.

En este contexto, Vera et al. (2019), mencionan que la ética debe ser parte de la formación y práctica profesional del psicólogo, los cuales deben estar basados en el respeto y principios deontológicos como la "fidelidad al cliente y lealtad por la profesión, veracidad y honestidad, confidencialidad, no maleficencia, beneficencia, autonomía y justicia" (p. 139). En México, la Sociedad Mexicana de Psicología (SMP) creó en 1999 la Comisión Nacional de Ética en Psicología (CONAEP) con el objetivo de vigilar y atender las denuncias que la ciudadanía en general y los propios colegas realizan sobre aspectos éticos y comportamentales de psicólogos que no llevan en su práctica profesional los lineamientos establecidos en el código ético.

Algunas de las consideraciones éticas referentes a la calidad de las intervenciones psicológicas, por la cual deben regir sus prácticas profesionales los psicólogos establecidas por la Sociedad Mexicana de 
Psicología, en el Código Ético del Psicólogo (2009, p. 26) son las siguientes:

Art. 29. En el que se habla que, al efectuar intervenciones terapéuticas preventivas, educativas, organizacionales, sociales, consejo psicológico directo, por escrito, radio, teléfono, televisión, internet o cualquier otro medio de comunicación, el psicólogo deberá basarse en la investigación y datos de la eficacia. En este artículo, se reafirma que el profesional que ofrece los servicios de telepsicología, debe capacitarse e intervenir desde una formación basada en evidencia y no conducirse de manera empírica, independientemente del medio de comunicación que considere pertinente.

En el art. 30 se menciona que el psicólogo deberá efectuar solamente intervenciones para las cuales posee educación, formación o experiencia supervisada y la pericia necesaria. El profesional debe presentar sus acreditaciones que lo avalan como una persona competente en el ejercicio de su profesión.

El art. 32 hace referencia a que el psicólogo deberá identificar los casos para los que algunas técnicas o procedimientos de intervención no sean aplicables o requieran ajustes ya sea por raza, género, idioma o nivel socioeconómico. En el caso de la telepsicología se deberá tener muy en cuenta el aspecto multicultural ya que este tipo de servicio permite la posibilidad de atender diferentes zonas y contextos geográficos, en la que es necesario adaptar la intervención que se esté llevando a cabo.

El art. 35 señala que el psicólogo que hace uso de medios automatizados a distancia, como es el caso de la telepsicología, debe estar alerta a la comunicación no verbal, debido a que es complicado identificar las expresiones emocionales que llegan a contradecir o a reafirmar el discurso, convirtiéndose en una de las limitantes de este servicio a diferencia de la atención cara a cara.

Asimismo, la SMP en el apartado D del Código Ético, sobre Responsabilidad hacia y la humanidad hace enfatiza los principios básicos que rigen los comportamientos de los psicólogos y que estipula que siempre deberá promover el bienestar hacia la sociedad y la humanidad en general sin distingos, cumpliendo con los principios éticos y basados en una psicología científica que permita dar resultados benéficos para quien requiere el servicio de la telepsicología.

También la Federación Nacional de Colegios, Sociedades y Asociaciones de Psicólogos de México A.C. (Federación Nacional de Colegios, 2018) establece el "Código de ética de las y los psicólogos mexicanos". Este código tiene la finalidad de orientar las prácticas y conductas profesionales en los diferentes ámbitos laborales de la psicología, resaltando la falta de conocimiento o una inadecuada comprensión, de los lineamientos éticos no lo exime de responsabilidad ante una acusación del ejercicio de su práctica.

Por último, con el objetivo de mejorar el debate acerca de las cuestiones éticas de la provisión de psicoterapia a distancia a través de la tecnología, Stoll et al. (2020) realizaron una revisión profunda de los principales argumentos éticos a favor y en contra de las distintas formas 
de psicoterapia en línea. Los resultados reflejaron 24 argumentos éticos en pro de la psicoterapia en línea y 32 en contra.

Algunos argumentos en pro de la telepsicología fueron el mayor acceso a la psicoterapia, y la disponibilidad y flexibilidad del servicio, mejor comunicación y beneficios de la terapia, ventajas sobre características individuales del cliente como la ubicación, conveniencia, satisfacción, aceptación y más demanda y ventajas económicas.

Los cinco argumentos en contra fueron aspectos de privacidad, seguridad y confidencialidad, la competencia del psicólogo y necesidad de capacitación especializada, los Obstáculos en la comunicación a causa de la tecnología, lagunas en la investigación y problemas de emergencia.

\section{Mindfulness y psicoterapia de tercera generación}

Las psicoterapias de tercera generación se centran en la aceptación, conciencia plena o mindfulness, desactivación cognitiva, dialéctica, valores, espiritualidad y las relaciones (Hayes et al., 1999). Sin intentar modificar las cogniciones, se centran en la función de los eventos para la vida e intervienen alterando los contextos verbales. Ningún pensamiento, sentimiento o recuerdo es patológico o disfuncional, sino que dependerá de su función en el contexto y puede reencuadrarse (Hayes et al., 1999). Se utilizan técnicas tales como ejercicios experienciales, metáforas, paradojas y cuentos hasta que el paciente se da cuenta de que intentar controlar las cosas forma parte del problema (Mañas, 2006).

Mindfulness es traducido como atención y conciencia plena, o presencia atenta y reflexiva. Se busca que el paciente se centre en el momento presente de un modo activo, procurando no interferir, ni valorar, lo que siente o percibe en cada momento. Como procedimiento terapéutico busca que las emociones y las cogniciones se acepten sin intentar ser modificados, observándose a sí mismo desde la compasión y es una habilidad que puede ser entrenada (Kabat-Zinn, 1994), experimentando las sensaciones corporales haciendo body scan, con la respiración o la imaginación, que con disciplina tiene efectos positivos en el funcionamiento ordinario de la persona (Kabat-Zinn, 2002).

\section{Evaluación de las intervenciones psicológicas}

En los últimos años, la satisfacción de consumidores y usuarios con los servicios es un tema que cada vez ha ido adquiriendo más importancia hasta llegar también al campo de la psicoterapia. La evaluación de la psicoterapia ha sido medida por instrumentos encaminados a valorar el nivel de cambio en relación con los síntomas, la calidad de vida, el funcionamiento psicosocial, nivel cognitivo, aspectos conductuales, etc., asimismo se han usado procesos de control de calidad y de valoración del grado de satisfacción de los usuarios de servicios de salud en general. Sin embargo, el grado de satisfacción con el servicio psicoterapéutico recibido, 
tanto en la práctica clínica como en la investigación es un tema poco estudiado.

Específicamente, en Latinoamérica son escasos los estudios sobre satisfacción con servicios de atención psicológica y salud mental. Monitorear y conocer la satisfacción de los clientes en cuanto a los servicios de salud permite dar el seguimiento oportuno y detectar carencias para diseñar estrategias de acción adecuadas y orientadas a las necesidades y realidades de quienes son atendidos (Rydel, 2015). Dentro de los estudios encontrados para investigar el impacto de las intervenciones psicológicas realizadas, está la "Evaluación de la Satisfacción con la Atención Psicológica en el primer nivel de atención, basado en el autoreporte de los usuarios". Este estudio fue hecho en Uruguay por Déborah Rydel (2015) con objetivo de investigar el impacto de las intervenciones psicológicas realizadas en servicios del Primer Nivel de Atención pertenecientes a la Administración de Servicios de Salud del Estado en la satisfacción con el servicio, el bienestar psicológico, el bienestar general y la calidad de vida de los usuarios. Los resultados indicaron que existe un alto grado de satisfacción con la atención recibida al igual que el enfoque de satisfacción de usuarios es un proceso factible y válido que sirve como indicador de que los servicios brindados son de buena calidad.

En la Universidad Autónoma de Nayarit (UAN), la atención telepsicológica se ofrece como parte del servicio de pruebas diagnósticas de COVID-19, por medio del Laboratorio Nacional para Investigación en Inocuidad Alimentaria (LANIIA) (Express, 2020). Este laboratorio es parte del Centro Nayarita de Innovación y Transferencia de Tecnología (CENIT2) de la UAN y actualmente está aprobado por el Instituto Diagnóstico y Referencia Epidemiológicos (InDRE) para realizar la aplicación de la prueba oral y nasal llamada qRT-PCR que detecta moléculas del SARS-CoV-2 a través de una muestra parecida a un exudado faríngeo usando tecnología de avanzada y avalada por la Organización Mundial de la Salud (Express, 2020).

Derivado de lo anterior, se considera pertinente realizar estudios que valoren la opinión de los usuarios en cuanto al uso de la telepsicología, para que de esta manera se pueda determinar qué tan oportuno es continuar con su práctica, cómo se sienten los usuarios con esta nueva modalidad y tener las bases para establecer qué tanto les ayuda a las personas con la resolución de su problemática. Es por eso que en el presente trabajo se busca dar respuesta a lo anteriormente expuesto. Para ello se aplicó, a las personas positivas de COVID-19 derivadas del LANIIA, que reciben seguimiento telepsicológico, la escala de satisfacción con el tratamiento recibido (CRES-4). La CRES-4 mide la satisfacción del cliente en cuanto la solución del problema y la percepción del cambio emocional de acuerdo al estado emocional actual y el estado emocional pretratamiento. De esta manera, se tiene el objetivo de analizar el estado de satisfacción de los usuarios para poder así detectar áreas de oportunidad que permitan establecer pautas de acción y optimizar el servicio otorgado, si así se requiriera, al igual que considerar aplicar esta actual forma de atención no 
sólo en tiempos de COVID sino en la cotidianeidad de la profesión del psicoterapeuta.

\section{Método}

\section{Diseño}

El estudio se llevó a cabo bajo el enfoque cuantitativo de investigación, con un diseño preexperimental de un solo grupo con una sola medida post tratamiento (Hernández \& Mendoza, 2018).

\section{Participantes}

El universo del estudio lo constituyeron 86 pacientes nayaritas de un laboratorio, atendidos en el Filtro Universitario Basado en la Detección Molecular del Virus SARS-CoV-2 entre agosto y septiembre del 2020. Dichos pacientes fueron referidos al servicio de telepsicología ofrecido por la Universidad Autónoma de Nayarit a través de la directora del Servicio de Atención Telepsicológica.

Se les contactó y dio seguimiento telefónico a través de llamadas y mensajes de Whatsapp, después de haber sido informados de ser positivos a Covid-19. Se obtuvo una muestra probabilística de 61 pacientes seleccionados de forma aleatoria, de los cuales 41 eran mujeres y 20 hombres, cuyas edades oscilaron entre los 16 y 67 años $(\mathrm{ME}=36$; $\mathrm{DE}=8.13)$.

\section{Instrumentos}

\section{Escala de satisfacción con el tratamiento recibido (CRES-4)}

Originalmente la escala fue desarrollada por la empresa Consumer Reports (CR), fue adaptado posteriormente por Nielsen et al. (2004) y traducida y corregida por Feixas et al. (2012). Está constituida por cuatro reactivos que abordan la satisfacción, el grado de resolución del problema y el estado emocional previo y posterior al tratamiento $(\alpha=.63)$. Con base en los cuatro ítems, se obtienen tres componentes y una puntuación global.

El primer ítem es ¿qué tan satisfecho se encuentra con la forma en que su psicoterapeuta abordó el problema? El segundo, ¿en qué medida le ayudó el tratamiento, con relación al problema que consultó?, seguido por ¿cuál era su estado emocional cuando empezó el tratamiento? Para finalizar con ¿cuál es su estado emocional en este momento?

Aunque tiene un formato de respuesta tipo Likert con cinco y seis opciones de respuesta, se utiliza la fórmula [CRES4 $=(20 \times$ Satisfacción $)$ $+(20 \times$ Solución del problema $)+[12.5(4+$ Estado emocional actual Estado emocional pretratamiento)]. 
El máximo por cada componente es 100 y de la escala global, 300, cuanto mayor es la puntuación, mayor el grado de satisfacción del paciente.

\section{Procedimiento}

Fase 1: Cita del laboratorio en el Filtro Universitario Basado en la Detección Molecular del Virus SARS-CoV-2, para toma de muestra para diagnóstico de Covid-19, a través de la prueba qRT-PCR, además, se realiza una grabación de sonido de la tos del participante y firma de consentimiento informado donde se describe el proceso, se garantiza el anonimato y se solicita la participación voluntaria para diversas investigaciones de carácter biológico y psicológico.

Fase 2: Los resultados positivos de la comunidad universitaria son enviados por correo a la psicóloga que dirige el centro de atención telepsicológica de la Universidad cuyo perfil es sistémico estratégico, para su comunicación vía telefónica, dando inicio al servicio de atención telepsicológica, posterior a lo cual, el resultado laboratorial se envía por escrito a los pacientes a través de su correo electrónico. El servicio telepsicológico consta de comunicaciones diarias de la psicóloga, para evaluar el estado emocional del paciente, a través de whatsapp y de ser necesario, llamada telefónica.

Fase 3: A los 21 días, se les toma una segunda muestra a los pacientes para comprobar su diagnóstico y de ser negativo, se establece una última comunicación desde el servicio de telepsicología y se les ofrece la posibilidad de obtener un proceso telepsicológico cognitivo conductual breve, a través de alguno de los 67 psicoterapeutas voluntarios del SATEPUAN. Se Aplicó la Escala CRES-4 a la muestra de pacientes dados de alta seleccionados al azar.

\section{Análisis de datos}

Se utilizaron medidas de tendencia central y de dispersión para identificar las puntuaciones en cada dimensión y en la evaluación general. Posteriormente, con la intención de comparar la satisfacción con el tratamiento entre hombres y mujeres, se abordó el tipo de distribución de las variables para detectar el tipo de prueba estadística necesaria. Al no encontrarse una distribución semejante a la normal $(\mathrm{p}<.05$ en la prueba de Kolmogorov), se utilizaron herramientas no paramétricas en tal comparación, a saber, un conjunto de pruebas U de Mann Whitney. Finalmente se hizo una comparación sobre el estado emocional previo y posterior de los pacientes mediante la prueba de rangos de Wilcoxon. Tanto en la comparación por sexo como en el estado emocional, se agregó la prueba r de Rosenthal para la estimación del tamaño del efecto (Rosenthal, 1991). 


\section{Consideraciones éticas}

Se les presentó un formato de consentimiento informado, donde se informó a los participantes sobre los objetivos de la investigación, el tratamiento que se daría a los datos, con base al Reglamento de la Ley General de Salud en Materia de Investigación para la Salud (2020, capítulo I), desde el momento de la toma de muestras biológicas.

\section{Resultados}

\section{Resultados descriptivos}

En primera instancia, se calcularon medias y desviaciones estándar de la puntuación global y los factores de la Escala de satisfacción con el tratamiento recibido. La Tabla 1 muestra tales hallazgos.

Tabla 1

Tabla 1

Resultados generales

\begin{tabular}{|c|c|c|c|c|}
\hline Dimensiones & Mínimo & Máximo & ME & $D E$ \\
\hline Puntuación & 70 & 95.83 & 81.88 & 17.969 \\
\hline Satisfacción & 80 & 100 & 98.69 & 4.992 \\
\hline Solución del problema & 60 & 100 & 84.26 & 11.024 \\
\hline Percepción del cambio emocional & 50 & 87.5 & 62.70 & 11.746 \\
\hline Puntuación global & 190 & 287.50 & 245.65 & 17.969 \\
\hline
\end{tabular}

Fuente: Elaboración propia.

Comenzando por la dimensión de satisfacción, se puede apreciar que la puntuación obtenida fue bastante alta $(\mathrm{ME}=98.69$; $\mathrm{DE}=4.992)$, si se considera su cercanía con la calificación máxima posible (100). Enseguida, en la dimensión de Solución del problema, aunque fue ligeramente más baja que en el factor anterior, su media se encontró en un nivel alto $(\mathrm{ME}=84.26 ; \mathrm{DE}=11.024)$, indicando que, en general, los pacientes consideraron que el tratamiento recibido propició la resolución de su motivo de consulta.

Por otro lado, el cambio emocional, obtenido al contrastar subjetivamente el estado emocional previo y posterior al tratamiento, mostró el nivel más bajo ( $\mathrm{ME}=62.70 ; \mathrm{DE}=11.746)$. No obstante, es preciso tomar en cuenta que esta dimensión se califica un tanto diferente. Particularmente, hay un punto de corte en el nivel 50 y las puntuaciones por debajo de este son sinónimo de empeoramiento, es decir, que el paciente considera que su estado emocional es más negativo que al iniciar el tratamiento. En cambio, las puntaciones por encima de tal valor indicarán una mejoría. Sin embargo, los valores serán más altos 
en la medida en que el paciente haya llegado con un estado emocional sumamente negativo y al momento de terminar muestre valores muy positivos. Cuando el paciente no llega en un estado emocional tan deteriorado, pero aún así mejora, los resultados se muestran con niveles superiores al valor de referencia, pero no tan cercanos al valor máximo, como sucedió en este caso. Para profundizar sobre esta dimensión, se presenta en el siguiente apartado una comparación entre los niveles previos y posteriores del estado emocional.

Por último, se puede observar que hay una puntuación global alta ( $\mathrm{ME}=245.65 ; \mathrm{DE}=17.969)$, indicando que la evaluación de los pacientes sobre el tratamiento psicológico mediante telepsicología es satisfactoria. La Figura 1 muestra un gráfico con las puntuaciones.

\section{Figura 1}

\section{Resultados generales}

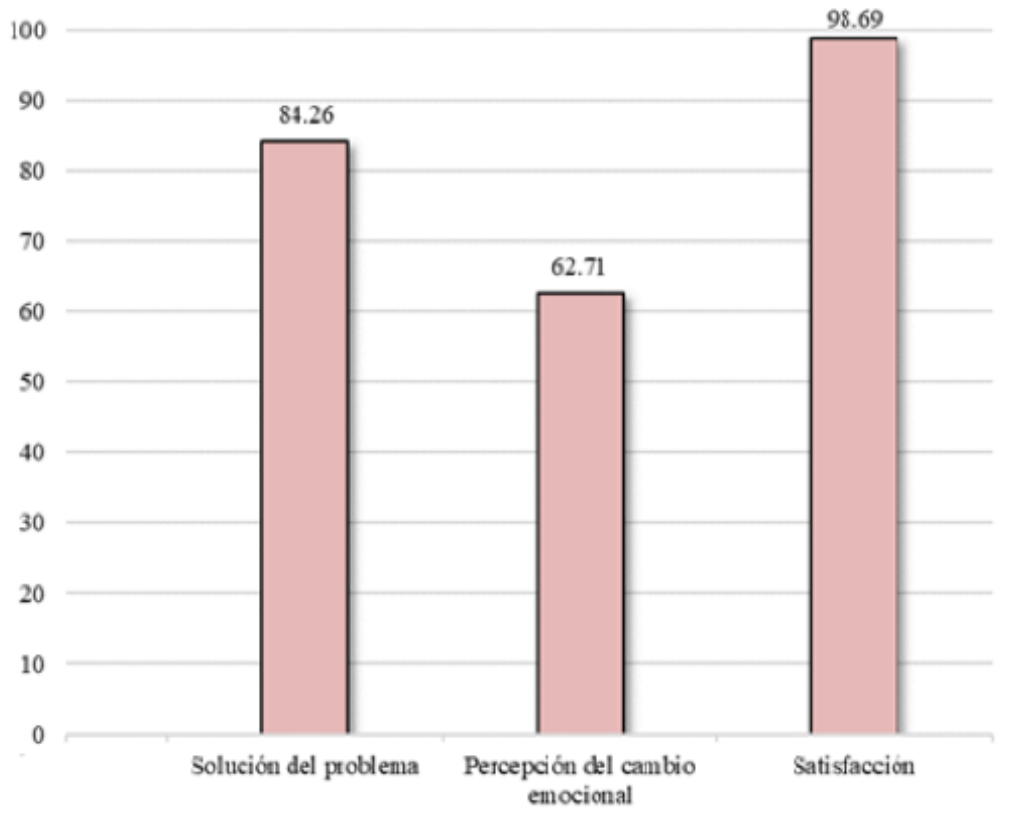

Fuente: Elaboración propia.

Figura 1

\section{Resultados inferenciales}

Como siguiente parte del análisis se realizó una comparación según el sexo de los pacientes para identificar diferencias en los niveles de satisfacción con el tratamiento entre hombres y mujeres. Los resultados se muestran en la Tabla 2. 
Tabla 2

Tabla 2

Comparación entre bombres y mujeres

\begin{tabular}{|c|c|c|c|c|c|c|c|}
\hline \multirow{2}{*}{ Dimensiones } & \multicolumn{2}{|l|}{ Mujeres } & \multicolumn{2}{|c|}{ Hombres } & \multirow{2}{*}{$Z$} & \multirow{2}{*}{$p$} & \multirow{2}{*}{$r$} \\
\hline & $M E$ & $D E$ & $M E$ & $D E$ & & & \\
\hline Satisfacción & 98.18 & 5.839 & 99.29 & 3.780 & -0.861 & 0.389 & 0.11 \\
\hline $\begin{array}{l}\text { Solución } \\
\text { problema }\end{array}$ & 84.24 & 11.997 & 84.29 & 9.974 & -0.078 & 0.938 & 0.01 \\
\hline $\begin{array}{l}\text { Percepción del } \\
\text { cambio emocional }\end{array}$ & 63.258 & 12.0786 & 62.054 & 11.5280 & -0.404 & 0.686 & 0.05 \\
\hline Puntuación global & 245.682 & 19.3273 & 245.625 & 16.5779 & -0.125 & 0.901 & 0.02 \\
\hline
\end{tabular}

Fuente: Elaboración propia.

A través de un conjunto de pruebas $U$ de Mann Whitney (considerando la ausencia de normalidad) no se encontraron diferencias estadísticamente significativas entre hombres y mujeres, constatando altos niveles de satisfacción para ambos grupos. La Figura 2 ilustra los promedios en cada una de las áreas.

Figura 2

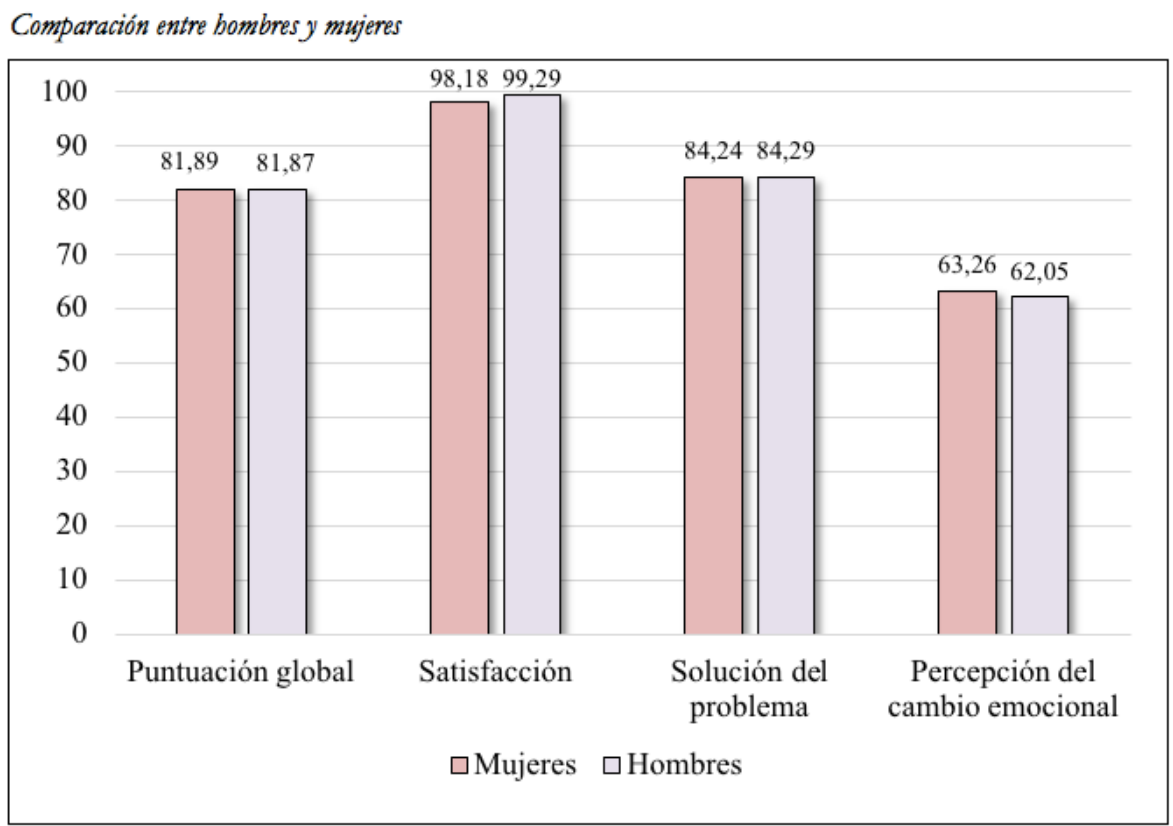

Fuente: Elaboración propia.

Figura 2

Como parte final del análisis de datos, se realizó un contraste entre las puntuaciones previas y posteriores de los pacientes sobre el estado emocional. Aunque ambas puntuaciones son necesarias para el cálculo de la dimensión de Percepción de cambio emocional, un contraste entre 
ellas permitirá detectar si el tratamiento de telepsicología influyen en la mejora del estado emocional de los pacientes. En la Tabla 3 se muestran los resultados del contraste.

\section{Tabla 3}

Tabla 3

Comparación entre el estado emocional previo y posterior

\begin{tabular}{|c|c|c|c|c|c|c|}
\hline \multicolumn{2}{|c|}{ Estado emocional previo } & \multicolumn{2}{|c|}{ Estado emocional actual } & \multirow{2}{*}{$Z$} & \multirow{2}{*}{$p$} & \multirow{2}{*}{$r$} \\
\hline$M E$ & $D E$ & $M E$ & $D E$ & & & \\
\hline 2.36 & 1.252 & 3.38 & .662 & -5.573 & .000 & .50 \\
\hline
\end{tabular}

Fuente: Elaboración propia

En este caso, las calificaciones en cada pregunta podrían fluctuar entre 0 y 4; puntajes altos indican un mejor estado emocional. En este sentido, la medición previa se encontró en un nivel intermedio $(\mathrm{ME}=2.36 ; \mathrm{DE}=1.252)$, mientras que el estado emocional posterior mejoró considerablemente $(\mathrm{M}=3.38 ; \mathrm{DE}=.662)$, estando más cerca del valor máximo posible. Además, la prueba de rangos de Wilcoxon confirmo la presencia de diferencias significativas entre las mediciones, con una magnitud o tamaño del efecto grande $(Z=-5.573 ; \mathrm{p}=.000 ; \mathrm{r}=.50)$. En la Figura 3 se pueden apreciar las diferencias citadas.

Figura 3

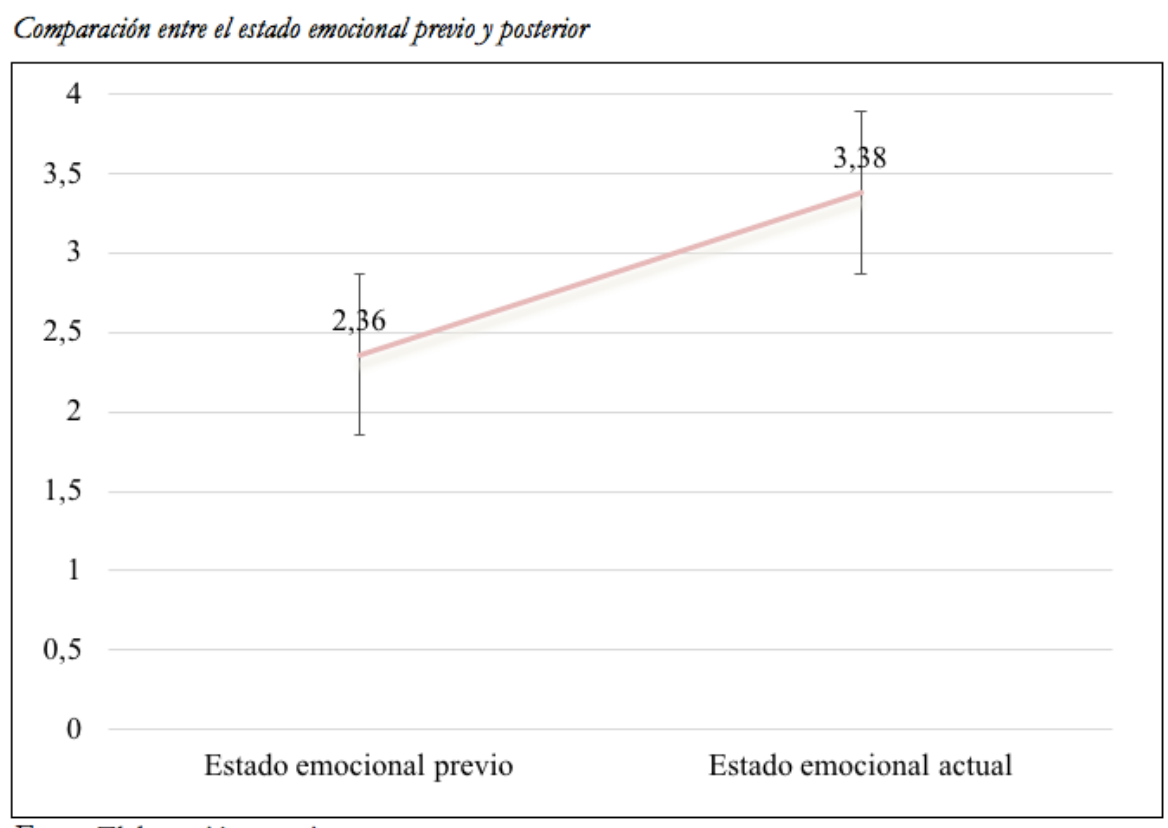

Fuente: Elaboración propia

Figura 3 


\section{Discusión}

Debido a la pandemia del COVID-19 se ha visto un incremento en el uso de servicios de telepsicología, reportándose que los profesionales de salud mental la han implementado en su práctica diaria (Feijt et al., 2020), y se ha descrito que el uso de servicios de salud mental brindados vía remota, ha facilitado el desarrollo e implementación de intervenciones en la emergencia presentada (Liu et al., 2020). La intervención implementada en esta investigación surgió como respuesta a la emergencia presentada y como un primer acercamiento conocer la experiencia del usuario. Se ha reportado la efectividad de la utilización de servicios de telepsicología. En un meta-análisis realizado con 65 artículos sobre el uso de psicoterapia por videoconferencia, se concluyó que es factible, que es adaptable a diversos formatos de psicoterapia, en diferentes tipos de población y que se ha asociado a buenos niveles de satisfacción por parte del usuario, así como con resultados clínicos similares a los obtenidos en consulta presencial (Backhaus et al., 2012).

En el presente estudio se tuvo como objetivo evaluar la experiencia del usuario de la atención telepsicológica del laboratorio de detección del virus SARS-COV2, incluyendo satisfacción al tratamiento recibido, la solución del problema y percepción del cambio emocional de acuerdo al estado emocional actual en comparación con el previo al tratamiento. $\mathrm{Al}$ igual que los resultados obtenidos en el meta-análisis mencionado (Backhaus et al., 2012), se presentaron buenos niveles de satisfacción con el servicio. Además, este servicio de telepsicología fue percibido como adecuado para solucionar el problema y se percibieron cambios positivos en el estado emocional, esto podría compararse con los resultados clínicos también mencionados en el meta-análisis (Backhaus, et al., 2012). Sin embargo, se muestra una limitación pues la variable "percepción de cambios en estado emocional” es subjetiva, sería más objetivo tener los resultados de las evaluaciones iniciales de los usuarios y los posteriores a la intervención para realmente determinar si se presentaron cambios clínicos.

Algunas ventajas percibidas sobre el uso de la telepsicología, que han sido reportadas en la literatura, entre ellas, que es eficiente y conveniente, que promueve un buen contacto con el cliente y que da información adicional sobre el ambiente del cliente (Feijt et al., 2020). Como primer acercamiento, y para los objetivos del estudio, que eran conocer la experiencia del usuario, ésta se reportó como adecuada, y que le generaba la percepción de solucionar el problema por el que se buscaba la ayuda.

La telepsicología puede ser una opción viable para tratar aspectos relacionados con la salud mental, siempre y cuando los proveedores del servicio estén preparados para ello (Acero et al., 2020; Gamble et al., 2015; McCord et al., 2020). Una de las sugerencias para futuros estudios deriva de este punto, y se refiere a incluir habilidades, satisfacción y experiencia del terapeuta ya que el presente estudio se enfocó en la experiencia del usuario. Otra sugerencia es que se evaluaran las necesidades y dificultades con el uso de la telepsicología, algunas de las que han sido reportadas 
son dificultades en la infraestructura y recursos tecnológicos, falta de procedimientos organizacionales, y poca factibilidad por parte de los clientes (Feijt et al., 2020). Tomando esto en cuenta, es probable que exista un sesgo en la investigación por el tipo de población que tiene acceso a la tecnología en comparación con la que no lo tiene, por ejemplo, personas que no tienen acceso a servicios de internet, a equipo de cómputo adecuado, personas que no están familiarizadas con la tecnología, etc. Se sugiere incluir las dificultades que se presentan en la población estudiada y recomendaciones para enfrentarlas en la práctica.

A pesar de haber obtenido resultados generales favorables, se pueden encontrar algunas limitaciones, la primera, el hecho de que la investigación está condicionada a las características inherentes a un estudio pre experimental. Sería esperable que la mejoría en el estado emocional se diera con el simple hecho del paso del tiempo sobre todo porque se espera una adaptación al diagnóstico o incluso remisión de síntomas de COVID-19 y que la mejoría emocional se debiera a esto, por ello sugerimos utilizar un grupo control con la intención de disminuir este sesgo. De igual manera, la forma de medir los cambios en el estado emocional es subjetiva, por lo que se sugiere incluir evaluaciones con instrumentos adaptados para este fin que tengan propiedades psicométricas adecuadas. Otra limitación tiene que ver con la muestra del estudio, en donde incluyeron personas que pudieron acceder al servicio, habría que ampliarla con personas que no tuvieran familiarización con la tecnología, o acceso a servicios de internet para ser usuarios de la intervención telepsicológica.

Por último, retomando las recomendaciones de la ONU (2020) mencionadas en la introducción del presente artículo sobre la minimización de los efectos de la pandemia en la salud mental, acorde a los resultados obtenidos, el uso de la telepsicología nos permite apoyar a la respuesta al COVID-19 creando un servicio de salud mental específico con ese propósito, que brinda atención oportuna, garantizando la disponibilidad de apoyo psicosocial y en materia de salud mental de emergencia. Faltaría el enfoque que abarque a toda la sociedad para promover, proteger y cuidar la salud mental; esto debido a la limitación sobre el acceso a tecnología y uso de internet en toda la población, sin embargo, nos ayuda a cubrir una mayor parte de la población que los servicios puramente presenciales.

Sería pertinente realizar más investigaciones sobre el tema de telepsicología dado la situación de la pandemia actual y la utilización de estos servicios por parte de diferentes profesionales, y que en Latinoamérica son escasos los estudios sobre satisfacción de servicios de atención psicológica y salud mental. Monitorear y conocer la satisfacción de los clientes en cuanto a los servicios de salud permite dar el seguimiento oportuno y detectar carencias para diseñar estrategias de acción adecuadas y orientadas a las necesidades y realidades de quienes son atendidos. 


\section{Conclusión}

De acuerdo con los resultados, se puede concluir que la atención telepsicológica brindada por el laboratorio de detección de SARSCOV-2, es evaluada de manera positiva por los pacientes, teniendo altas puntuaciones en las dimensiones "satisfacción" y "solución del problema".

Lo anterior podría indicar que, en general, los pacientes consideraron que el tratamiento recibido propició la resolución de su motivo de consulta. El contraste en la percepción del estado emocional de los pacientes sugiere que el tratamiento de telepsicología influye efectivamente en la mejora de su estado emocional.

El éxito de la telepsicología, evidenciado en éste y otros estudios, podría ser un parteaguas en los esquemas típicos de atención psicoterapéutica en México, durante y después de la pandemia.

\section{Referencias}

Acero, P., Cabas, K., Caycedo, C., Figueroa, P., Patrick, G., \& Rudas, M. M. (2020). Telepsicología: sugerencias para la formación y el desempeño profesional responsable. Colpsic. https://ascofapsi.org.co/pdf/Libros/Tele psicologia_web.pdf

Argüero-Fonseca, A., Cervantes-Luna, B. S., Martínez-Soto, J., Alva-Rangel, N. T., De Santos-Ávila, F., Espinosa-Parra, I. M., Parra-Jiménez, E. L., Aguirre-Ojeda, D. P., Gómez-Gloria, J., Díaz-Flores, D. P., López-Beltrán, I., \& Bautista-Moedano, R. M. (2020). Guia de Atención Psicológica Virtual para personas de grupos vulnerables en crisis por la pandemia COVID-19. Universidad Autónoma de Nayarit. http://dspace.uan.mx:8 080/handle/123456789/2335

Argüero-Fonseca, A., Cervantes-Luna, B. S., Martínez-Soto, J., de SantosÁvila, F., Aguirre-Ojeda, D. P., Espinosa-Parra, I. M., \& Beltrán, I. L. (2021). Telepsicología en la pandemia Covid-19: una revisión sistemática. Uaricha, 18, 1-10.

American Psychological Association. (2013). Guidelines for the practice of telepsychology. American Psychologist, 68(9), 791-800. http://dx.doi.org/ 10.1037/a0035001.

Backhaus, A., Agha, Z., Maglione, M. L., Repp, A., Ross, B., Zuest, D., \& Thorp, S. R. (2012). Videoconferencing psychotherapy: A systematic review. Psychological Services, 9(2), 111-131. https://doi.org/10.1037/a0027924

Balluerka, M., Gómez, J., Hidalgo, M., Gorostiaga, M., Espada, J., Padilla J., \& Santed, M. (2020). Las consecuencias psicológicas de la COVID-19 y el confinamiento. Informe de investigación. Publicaciones de la Universidad del País Vasco. https://addi.ehu.eus/bitstream/handle/10810/45924/Consecue ncias\%20psicol\%C3\%B3gicas\%20COVID-19\%20PR3\%20DIG.pdf?seq uence $=1 \&$ isAllowed $=\mathrm{y}$.

Burgoyne, N., \& Cohn, A. S. (2020). Lessons from the transition to relational teletherapy during COVID - 19. Family Process, 59(3), 974-988. https://d oi.org/10.1111/famp.12589 
Centro de Psicología Aplicada de la Universidad Autónoma de Madrid en Colaboración con el Colegio Oficial de Psicólogos de Madrid. (2018). Guia para la Intervención Telepsicológica. https://www.psichat.es/guia-pa ra-la-intervencion-telepsicologica-2019.pdf

Colegio Oficial de Psicólogos de Madrid. (2017). Uso de las nuevas tecnologías por parte de los psicólogos españoles y sus necesidades. Clínica y Salud, 28(2), 81-91. http://dx.doi.org/10.1016/j.clysa.2017.01.001

Consejo General de la Psicología de España. (2017). Guía para la práctica de la telepsicología. American Psychologist, 68(9). https://doi.org/10.23923/co pd.telepsicologia.2017

Comisión Nacional de Salud de las Personas de República de China. (2020, febrero 13). Mental health services deployed across China in fight against epidemic. http://en.nhc.gov.cn/2020-02/13/c_76516.htm

De la Torre, M., \& Pardo, R. (2018) Guía para la intervención telepsicológica. Colegio Oficial de Psicólogos de Madrid. https://www.psichat.es/guia-pa ra-la-intervencion-telepsicologica-2019.pdf

Express. (2020, mayo 5). Instala UAN laboratorio para prueba de COVID a universitarios. http://periodicoexpress.com.mx/instala-uan-laboratorio-p ara-prueba-de-covid-a-universitarios/

Federación Nacional de Colegios, Sociedades y Asociaciones de Psicólogos de México. (2018). Código de ética de las y los psicólogos mexicanos. http://ww w.fenapsime.org.mx/download/codet.pdf

Feixas, G., Pucurull, O., Roca, C., Paz, C., García-Grau, E., \& Bados, A. (2012). Escala de Satisfacción con el Tratamiento Recibido (CRES-4): la versión en español. Revista de Psicoterapia, 23(89), 51-58.

Feijt, M., de Kort, Y., Bongers, I., Bierbooms, J., Westerink, J., \& IJsselsteijn, W. (2020). Mental health care goes online: Practitioners' experiences of providing mental health care during the COVID-19 pandemic. Cyberpsychology, Behavior, and Social Networking, 23(12), 860-864. https ://doi.org/10.1089/cyber.2020.0370

Gamble, N., Boyle, C., \& Morris, Z. A. (2015). Ethical practice in telepsychology. Australian Psychologist, 50(4), 292-298. http://dx.doi.org /10.1111/ap.12133.

González, M., \& Sánchez, L. (2020). Atención psicológica en la crisis por Covid-19: exploración sobre limitaciones, posibilidades y retos. Revista Análisis de la Realidad Nacional, 9(187), 36-48. http://ipn.usac.edu.gt/w p-content/uploads/2020/07/IPN-RD-187.pdf.

Guerrero, L. (2020). Estrategias de intervención en crisis por pandemia de Coronavirus - Colombia 2020. Campo de la psicología en gestión del riesgo en emergencias y desastres. [Diapositivas de Power Point]. https://drive.go ogle.com/open?id=1mdBFGW2SfDVcxMM03xpmJlsoYQJD6aNj

Hayes, S. C., Strosahl, K. D., \& Wilson, K. G. (1999). Acceptance and commitment therapy. Guilford Press

Hernández, R., \& Mendoza, C. (2018). Metodología de la Investigación: las rutas cuantitativa, cualitativa y mixta. McGraw-Hill.

Kabat-Zinn, J. (2002). Guided mindfulness meditation: Body scan meditation. [Compact disc recording]. Stress Reduction CDs and Tapes. ISBN 1-59179-359-9. 
Kabat-Zinn, J. (1994). Wherever you go there you are: Mindfulness meditation in everyday life. Hyperion.

Larroy, C., Estupiña, F., Fernández-Arias, I., Hervás, G., Valiente, C., Gómez, M., Crespo, M., Rojo, N., Roldán, L., Vázquez, C., Ayuela, D., Lozano, B., Martínez-Bernardo, A., Pousada, T., Gómez, A., Asenjo, M., JoséRodrgigo, J., Florido, R., Vallejo-Achón, M., Sanz, S., Martos, L., González del Valle, S., Anton, A., Jiménez, A., De Miguel, A., Abarca, B., De la Torre, M. \& Pardo, R. (2020). Guia para el abordaje no presencial de las consecuencias psicológicas del brote epidémico de COVID-19 en la población general. https://www.copmadrid.org/web/comunicacion/noticias/1484 /guia-el-abordaje-no-presencial-las-consecuencias-psicologicas-brote-epi demico-covid19-la-poblacion-general

Liu, S., Yang, L., Zhang, C., Xiang, Y. T., Liu, Z., Hu, S., \& Zhang, B. (2020). Online mental health services in China during the COVID-19 outbreak. The Lancet Psychiatry, 7(4), e17-e18. https://doi.org/10.1016/S2215-03 66(20)30077-8

Mañas, I. (2006). Meditación y pensamiento: disolución y supervivencia del "Yo". Comunicación presentada en el 8th Internacional Congress on Behavior Studies. Santiago de Compostela, España. https://xdoc.mx/documents/ mindfulness-5c020cf824fb4

McCord, C., Bernhard, P., Walsh, M., Rosner, C., \& Console, K. (2020). A consolidated model for telepsychology practice. Education \& Training, 76(6),1060-1082. https://doi.org/10.1002/jclp.22954

McDonald, A., Eccles, J. A., Fallahkhair, S., \& Critchley, H. D. (2020). Online psychotherapy: Trailblazing digital healthcare. BJPsych Bulletin, 44(2), 60-66. https://doi.org/10.1192/bjb.2019.66

Nielsen, S., Smart, D., Isakson, R., Whorten, V., Gregersen, A., \& Lambert, M. (2004). The consumer reports effectiveness score: What did consumers report? Journal of Counseling Psychology, 51(1), 25-37. http://dx.doi.org/ 10.1037/0022-0167.51.1.25

Organización de las Naciones Unidas [ONU] (2020, 13 de mayo). Informe de politicas: La COVID-19 y la necesidad de actuar en relación con la salud mental. https://www.un.org/sites/un2.un.org/files/policy_brief_-_covid _and_mental_health_spanish.pdf

Organización Panamericana de la Salud [OPS]. (2020, 19 de junio). COVID-19 Intervenciones recomendadas en salud mental y apoyo psicosocial (SMAPS) durante la pandemia. https://www.paho.org/es/documentos/covid-19-intervencio nes-recomendadas-salud-mental-apoyo-psicosocial-smaps-durante

Organización Panamericana de la Salud (OPS) \& Organización Mundial de la Salud Oficina Regional para las Américas México (OMS). (s/f). México se encuentra en una situación "extremadamente compleja, por la pandemia de COVID-19, dice OPS. https://www.paho.org/mex/index.php?option=com_content已view $=a$ rticlejid=1544:mexico-se-encuentra-en-una-situacion-extremadamente-c ompleja-por-la-pandemia-de-covid-19-dice-opsé-Itemid =499

Organización Mundial de la Salud [OMS]. (2020, 5 de octubre). Los servicios de salud mental se están viendo perturbados por la COVID-19 en la mayoría de los paises, según un estudio de la 
OMS. https://www.who.int/es/news/item/05-10-2020-covid-19-disrup ting-mental-health-services-in-most-countries-who-survey

Organización Mundial de la Salud [OMS]. (2021, 8 de abril). Panel de la OMS sobre la enfermedad por coronavirus (COVID-19). https://covid19.who.i nt/

Pierce, B. S., Perrin, P. B., Tyler, C. M., McKee, G. B., \& Watson, J. D. (2021). The COVID-19 telepsychology revolution: A national study of pandemicbased changes in U.S. mental health care delivery. American Psychologist, 76(1), 14-25. https://doi.org/10.1037/amp0000722

Reglamento de la Ley General de Salud en materia de Investigación para la salud. (2020, October 9). http://www.salud.gob.mx/unidades/cdi/nom/compi /rlgsmis.html

Rosenthal, R. (1991). Meta-Analytic procedures for social research. Sage publications. https://dx.doi.org/10.4135/9781412984997

Rydel, D. (2015). Evaluación de la satisfacción con la atención psicológica en el primer nivel de atención: basado en el autoreporte de los usuarios. [Tesis de maestría]. Universidad de la República, Uruguay. https://hdl.handle.net/ 20.500.12008/8336

Secretaría de Salud, Álvarez. J., Andrade, C., Vieyra, V., Esquivias, H., Merlín, I., Neria, R., \& Bezanilla, J. (2020). Manual operativo del curso emergente para la brigada de atención psicoemocional y psicosocial a distancia durante la pandemia de la COVID-19 en México. Módulo 1. http://inprf.gob.mx/ ensenanzanew/archivos/2020/manual_brigadas_2020.pdf

Sociedad Mexicana de Psicología. (2009). Código ético de psicología. Trillas. http://www.psicologia.unam.mx/documentos/pdf/comite_etica /CODIGO_ETICO_SMP.pdf

Stoll, J., Müller, J. A., \& Trachsel, M. (2020). Ethical issues in online psychotherapy: A narrative review. Frontiers in Psychiatry, 10, 993. https ://doi.org/10.3389/fpsyt.2019.00993

Tullio, V., Perrone, G., Bilotta, C., Lanzarone, A., \& Argo, A. (2020). Psychological support and psychotherapy via digital devices in Covid-19 emergency time: Some critical issues. Medico-Legal Journal, 88(2), 73-76. https://doi.org/10.1177/0025817220926942

Vera, B., Castro, J., \& Caicedo, L. (2019). La intervención del psicólogo a través de las redes sociales: una perspectiva desde la ética.Didasc@lia:Didáctica y Educación, 10(2), 133-146. 